\title{
Ciudades intermedias: dinámicas de intermediación desde la noción de lugar. El caso de la región de la Araucanía, Chile $^{1}$
}

\author{
Gonzalo Salazar², Martín Fonck ${ }^{3}$ \\ y Luis Vergara ${ }^{4}$
}

\begin{abstract}
RESUMEN
El trabajo es una contribución al debate de las ciudades intermedias y sus dinámicas de intermediación. Si bien se reconocen como importantes los aspectos centrales de este debate, se cuestiona su tendencia a homogenizar las ciudades intermedias. Por el contrario, se plantea que es necesario comprender las ciudades intermedias desde las practicas socio-espaciales, en donde la definición de lugar asoma como elemental para entender las dinámicas de intermediación y la unicidad de cada una de estas ciudades. El área de estudio es la región de La Araucanía de Chile, específicamente las ciudades de Angol, Victoria y Villarrica. A partir de atributos claves de la condición de lugar propuestos por Massey (2012), se examinan empíricamente prácticas y tensiones socio-espaciales constitutivas de las dinámicas de intermediación. Este acercamiento aporta en una comprensión de las ciudades intermedias y sus respectivos procesos de intermediación de forma más apropiada y poco desarrollado en la literatura especializada.
\end{abstract}

Palabras clave: ciudades intermedias; lugar; intermediación; sustentabilidad urbana-territorial

\begin{abstract}
The following article contributes to the discussion of intermediate cities and their dynamics of intermediation. Although it is recognized the theoretical advancement generated in this field of research in the recent years, the article questions its tendency to homogenize the understanding of these types of cities. The article suggests that it is necessary to comprehend the intermediate cities from their constitutive social practices. From this perspective, the notion of place is introduced as a vital aspect to understand the process of intermediation and the particularity of each urban system. From some key constitutive aspects of the notion of place suggested by Massey (2012) the article empirically examines social practices and tensions that are part of the dynamics of intermediation in three case studies. It is argued that this conceptual approach contributes to the development of a more appropriate and relational understanding of intermediate cities and their respective dynamics of intermediation. The area of study is in the La Araucanía Region, specifically in the intermediate and intercultural cities of Villarrica, Angol and Victoria.
\end{abstract}

Keywords: intermediate cities, place, intermediation, urban sustainability.

Esta investigación ha sido financiada por los siguientes fondos públicos: Fondecyt Regular № 1181575, Proyecto CONICYT/FONDAP No15110020 (CEDEUS), Proyecto CONICYT/FONDAP No 15110006 (CIIR). Artículo recibido el 6 de junio de 2016, aceptado el 29 de marzo de 2017 y corregido el 30 de junio de 2017.

Centro de Desarrollo Urbano Sustentable (FONDAP - CEDEUS) / Centro de Estudios Interculturales y Indígenas (FONDAP - CIIR) / Centro UC de Desarrollo Local (CEDEL-UC) / Campus Villarrica, Instituto de Estudios Urbanos y Territoriales, Pontificia Universidad Católica de Chile (Chile). E-mail: gonzalosalazar@uc.cl

Centro de Estudios Interculturales y Indígenas (FONDAP - CIIR) / Centro UC de Desarrollo Local (CEDEL-UC), Pontificia Universidad Católica de Chile (Chile). E-mail: mfonck1@uc.cl

4 Doctorado en Arquitectura y Estudios Urbanos / Centro UC de Desarrollo Local (CEDEL-UC), Pontificia Universidad Católica de Chile (Chile). E-mail: Ilvergara@uc.cl 
La población que habita en asentamientos urbanos ha estado creciendo a nivel global y se espera que la tendencia continúe (UNFPA 2007; UN-Habitat 2010). Contrario a la creencia general, la mayor parte de la población urbana no reside en áreas metropolitanas, sino en asentamientos medios (UNFPA 2007). Al igual que en el contexto mundial, en Chile se pronostica que la población urbana continúe aumentando (Hidalgo 2002; De Mattos 1999; Hidalgo et al., 2009), especialmente en las ciudades consideradas intermedias (Borsdorf et al., 2012; Bellet y Beltrao 2009). Es en este contexto, como estos asentamientos no solo han adquirido gran relevancia en el sistema urbano nacional (Rojas et al., 2015; Canales y Canales 2013; Borsdorf et al., 2012; Rodríguez \& González, 2006), sino que también han captado el interés de una buena parte de los estudios urbanos y territoriales (ver, por ejemplo, Maturana et al. 2018; Maturana y Rojas 2015; Henríquez 2014; Canziani y Schejtman 2013; Borsdorf et al., 2012).

El esfuerzo de la literatura ha estado puesto en la caracterización de la ciudad intermedia. Se ha dicho que estos lugares son: a) centros de equilibrio y sustentabilidad territorial con el ecosistema circundante del que son parte; b) centros más gobernables y con una mayor participación ciudadana; c) centros de importancia administrativa, al contar frecuentemente con la administración del gobierno local o regional; d) centros sociales y de identificación del ciudadano con su ciudad, al tratarse de asentamientos con una escala y dimensión más humana; e) espacios urbanos menos segregados y con un mayor grado de integración social, por cuanto los espacios públicos tienden a ser compartidos por todos sus habitantes; f) lugares que se relacionan indistintamente con tres escalas territoriales, específicamente entre la ciudad y su hinterland rural, la ciudad y otros centros metropolitanos y la ciudad con dinámicas productivas globales; y g) ciudades culturalmente homogéneas con menor conflictividad social y más gobernables (ver Llop y Bellet, 2004; Bellet \& Beltrao, 2009; Bezerin, 2015; Skewes et al., 2012; Maturana \& Rojas, 2015; Da Silva, 2016).

En este trabajo se argumenta que, si bien los estudios y definición de ciudades intermedias han contribuido en sistematizar patrones comunes sobre los asentamientos de menor peso demográfico, han tendido a ofuscar las dinámicas y procesos locales, obviando las particularidades que estas ciudades tienen en la práctica. Si bien el concepto de ciudad intermedia entrega una aproximación general útil, es necesario profundizar en estos sistemas urbanos a partir de la experiencia de sus habitantes para comprender adecuadamente la diversidad y complejidad de procesos que las ciudades experimentan desde su carácter de lugares emplazados, y así poder aportar a una planificación urbana más inclusiva y sustentable. Desarrollamos este argumento, explorando tres de las características que la literatura ha atribuido a las ciudades intermedias: su condición de lugares integrados socialmente, sus relaciones con diferentes escalas territoriales y su condición de lugares culturalmente homogéneos.

Desde el punto de vista conceptual, la discusión respecto a cómo se ha entendido la ciudad intermedia se ha movido desde un criterio demográfico hacia uno relacional. El criterio demográfico ha definido a la ciudad media en términos de la cantidad de habitantes, poniendo el foco en aquellas que poseen entre 50 mil y 1 millón de habitantes en Latinoamérica (Jordan y Simioni, 1998). No obstante, dicho enfoque ha sido criticado, primero, por promover una definición estática de este tipo de ciudades que no ayuda en la comprensión de la naturaleza de las mismas (Bolay \& Rabinovich, 2004; Canziani y Schejtman, 2013), y segundo, por ser excesivamente dependiente y variable respecto de contextos específicos (Roberts, 2014). Lo que es una ciudad 
media en un país como Brasil, puede ser considerado una ciudad metropolitana en un país como Chile o Uruguay.

Diversos trabajos han propuesto un foco hacia las dinámicas y funciones que cumplen este tipo de ciudades (Bellet y Llop, 2004; Bellet y Beltrao, 2009; Bolay \& Rabinovich, 2004; Berdegué et al., 2011; Canziani y Schejtman, 2013; Henríquez, 2014; Henríquez y Arenas, 2016). Perspectivas que en una línea común proponen el paso desde un concepto de ciudad media hacia uno de intermedia (Bellet y Beltrao, 2009; Maturana, 2015), reemplazando la referencia al tamaño por las características relacionales que el concepto de intermediación propone. De este modo, la ciudad intermedia ya no se entiende solamente por su variable demográfica, sino sobre todo por las dinámicas relacionales que ésta establece con diferentes escalas territoriales.

Al pensar las problemáticas que actualmente enfrentan dichos sistemas urbanos es necesario desentrañar el carácter de intermediación que continuamente establecen con su territorio inmediato (Berdegué et al., 2015; Canziani y Schejtman, 2013). En esta línea Berdegué et al. (2011) han abordado la interacción urbano-rural de ciudad intermedia a través de los flujos de conmutación, resaltando, en una mirada exclusivamente espacial, los cortos tiempos de viaje que hay entre estas y su entorno. En el caso chileno, Canales y Canales (2013), han estudiado la relación de las ciudades intermedias con el entorno proponiendo el concepto de "agrópolis", el que resalta la conexión que estas tienen con las actividades económicas que se realizan en lo que tradicionalmente se denomina como espacio rural. Estos trabajos ilustran cómo esta articulación de territorios funcionales y/o ciudades intermedias se marca por un auge de ese tipo de asentamientos asociado a la apertura comercial de la región en base a explotación de recursos primarios (Portes y Roberts, 2005; Borsdorf et al., 2008).

Si bien la aproximación desde la intermediación entrega importantes elementos para comprender la ciudad intermedia en un sistema de interacciones territoriales de mayor complejidad, no se desarrollan enfoques para abordar estos asentamientos desde sus actores y complejidades. Esto implica una gran dificultad respecto a comprender los discursos, prácticas y representaciones de los actores en relación a cómo entienden y definen lo que son sus asentamientos. Este acercamiento conlleva que se tienda a generalizar y agrupar una amplia gama de ciudades dentro de la categoría intermedia, lo que consecutivamente comienza a desvanecer lo local y la especificidad de cada uno de estos asentamientos. Por lo tanto, al igual como sucede al entender lo local a partir de lo global (Escobar, 2000; Massey, 2012), la definición de ciudad intermedia como interfaz genérica entre lo metropolitano y lo rural tiende a desdibujar la naturaleza del lugar.

En ese plano, en el presente trabajo se plantea como necesario un entendimiento de la ciudad intermedia que permita comprenderla a través de la intermediación y de las experiencias y prácticas de los actores. Por lo tanto, busca avanzar en la comprensión de la ciudad intermedia mediante el reforzamiento de su condición de lugar-esto es, "lugarizando" la ciudad intermedia. Para ello, se plantean dos preguntas de investigación: ¿Cómo las prácticas socio-espaciales de los actores locales constituyen procesos particulares de intermediación en las ciudades intermedias? Lo que a un nivel conceptual implica entender ¿cómo el lugar es un aporte en la discusión de ciudades intermedias? 
Como casos de estudio, se toman tres ciudades ubicadas en la Región de La Araucanía, Chile: Angol, Villarrica y Victoria. Dichas ciudades pueden considerarse intermedias tanto por sus características demográficas, por su función dentro del sistema de asentamientos urbanos regional y la relación que poseen con el territorio que las rodea. No obstante, más allá de las características comunes, poseen particularidades que retroalimentan la necesidad de "lugarizar" la discusión sobre estas ciudades.

El artículo se organiza en cinco apartados, además de la introducción. En el siguiente se desarrolla la discusión respecto la importancia de situar la discusión sobre el lugar en el estudio de ciudades intermedias. Luego se presenta la estrategia metodológica desplegada, enfatizando el carácter cualitativo e inductivo de la misma. A continuación, se presentan los casos de estudio, argumentando la relevancia que tiene para los propósitos de este trabajo el estudio de Angol, Victoria y Villarrica. Luego se da cuenta de los resultados, los que fueron organizados en tres dimensiones para entender el lugar: morfología urbana y relaciones sociales, movilidad intercultural e interculturalidad. Finalmente, se presentan conclusiones, en donde se reafirma la necesidad de comprender las ciudades intermedias no solo desde una condición conceptual, sino también desde la comprensión espacio temporal que elaboran sus actores "lugarizando" la ciudad intermedia.

\section{Hacia la "lugarización" de la ciudad intermedia}

Las aproximaciones planteadas en la introducción dan cuenta que el debate reciente de la ciudad intermedia se encuentra estrechamente asociado al contexto actual de la expansión global del capitalismo, y debe ser comprendido como un esfuerzo por analizar las tendencias demográficas y funcionales de cierto tipo de asentamientos humanos en esta etapa. Esto no solo con un fin conceptual, sino también para, mediante la comprensión de estas ciudades, aportar hacia procesos de planificación más inclusivos y sustentables. En ese escenario, es fundamental entender la ciudad intermedia como un fenómeno propio, y no como una escala de asentamiento entre lo rural y lo metropolitano.

Considerando lo anterior, un análisis de la ciudad intermedia en función de lo metropolitano sufre las mismas limitaciones que atañen a las conceptualizaciones de lo local a partir de la globalización. Como señala Escobar (2000), el reciente frenesí de la globalización tiene una incidencia importante en la comprensión de la cultura, el conocimiento, la naturaleza y en la economía, o bien sobre la comprensión espacio-temporal planteada por Massey (1994). Por ejemplo, respecto a la distinción entre lo global y lo local, la asimetría entre ambas escalas tiende a entregar roles relativamente rígidos a cada entidad, y en buena medida lo local suele ser entendido como una entidad espacial que sigue el guión de la globalización, pero que a su vez es distinta de la entidad espacial global (Escobar, 2000). En ese sentido, Escobar plantea que "lo global está asociado al espacio, el capital, la historia y la acción humana mientras lo local, por el contrario, es vinculado al lugar, el trabajo y las tradiciones" (2000: 128). La compresión espacio temporal que entrega la ciudad intermedia, en términos conceptuales, vendría a representar las dinámicas de los asentamientos humanos que se han insertado en última etapa al capitalismo global, y que aún no pueden ser entendidos como entidades metropolitanas en donde lo global es un elemento constitutivo. Es la tensión entre las entidades urbanas metropolitanas, entendiendo éstas como las que poseen un alcance explícito con la escala global, y las entidades urbanas in- 
termedias, las cuales intermedian entre lo metropolitano y lo no urbano, la que en buena medida define lo que es la ciudad intermedia.

Esta comprensión espacio temporal de ciudad intermedia, en torno a lo no metropolitano, que se puede encontrar en múltiples trabajos (Bellet y Llop, 2004; Bellet y Beltrao, 2009; Berdegué et al., 2015), tiene su funcionalidad en los procesos que quieren evidenciar, y que sin duda cuentan con una sólida base empírica que los sustenta. No obstante, esta comprensión espacio temporal en el contexto del "frenesí de la globalización" tiende a desdibujar, o inclusive a hacer desaparecer, el lugar como tal" (Escobar, 2000). En el lugar radican, y por ende se pierde mediante la definición de local (en el marco global-local) o bien de ciudad intermedia, aquellos elementos constitutivos del conocimiento local o inclusive aquellos modelos culturales de naturaleza que pueden diferir de las lógicas capitalistas de producción (Escobar 2000). En la misma dirección, Massey (2012) plantea que la conceptualización espacio-temporal basada tanto en isomorfismos, como la del espacio de flujos propia de la globalización, tiende a anular las diferencias espaciales y les niega la posibilidad de autonomía. Esto quiere decir que una comprensión isomórfica y/o de flujos, como podría ser la de ciudad intermedia o lo local (desde la lógica global-local), anularía la posibilidad de comprender la particularidad de la comprensión espacio-temporal de los actores, y por ende de cómo se construye socialmente el espacio. En efecto, Lombard (2015) plantea la "lugarización" de los asentamientos informales como un ejercicio conceptual que permite disputar políticamente la distinción entre formal/informal, en tanto la construcción de lugar propia de esos asentamientos es social y política, por lo que la definición informal tendería a marginalizarlos y negarles la posibilidad de ser reconocidos como espacios comunes. En buena medida, la asimilación conceptual, política y práctica de los procesos de "lugarización" son gravitantes para las disputas sociales actuales (Escobar 2001)

Ese ejercicio conceptual, y de cierto modo también político, requiere en buena medida una extensión de las estructuras conceptuales y metodológicas que permita generar las comprensiones espacio-temporales derivadas de la multiplicidad de interacciones sociales que se dan en el espacio. En ese plano, categorías funcional y conceptualmente rígidas, como la ciudad intermedia, pueden entregar una aproximación a ciertas características que acontecen en el plano local, pero no la perspectiva ni la unicidad espacio temporal que construyen los actores, por lo cual es necesario abrirse a otros marcos que permitan identificar y analizar adecuadamente las interacciones. "Una vez que nos alejamos de los discursos de confrontación o relación entre lo global y lo local, aparece ante nosotros un panorama más complejo y libre en que más que conceptos como 'local' o 'global', nos servimos de conceptos más amplios y abiertos como 'espacio' o 'lugar' (González, 2005: s/p). De ese modo, se hace necesario avanzar hacia una comprensión espacio temporal donde se pueda apreciar la co-existencia de distintas narrativas, y que los lugares son articulaciones de relaciones sociales, que acontecen tanto dentro del lugar, como también a partir de interacciones más allá de éste (Massey 2012). Si bien el ejercicio de definir el lugar es complejo, Massey plantea que una definición progresista de lugar puede considerar cuatro aspectos. Primero, el lugar es fundamentalmente un proceso de interacciones sociales que se desarrollan en él. Segundo, las fronteras del lugar son un ejercicio operativo para su estudio, pero en la práctica no son necesarias para su conceptualización. Las interacciones sociales que atraviesan el lugar pueden darse tanto dentro como fuera de él. Tercero, los lugares no poseen identidades específicas, pues están llenos de conflictos internos que los configuran. Cuarto y final, ninguno de los aspectos antes señalados niega el lugar ni su unicidad. En efecto, la globalización lejos de homogeneizar lugares, es una fuente de unicidad de lugares. 
Tomar como referencia estos cuatro aspectos permite aproximarnos a la ciudad intermedia desde la escala de los actores y comprender los procesos locales desde su dimensión de lugar, consiguiendo, por lo tanto, "lugarizar" (Lombard, 2015; Massey, 2012) la categoría de ciudad intermedia. Desde esta aproximación el concepto de ciudad intermedia adquiere mayor sentido para la misma ciudad y las dinámicas vividas. En esta línea, enfocarse en las particularidades del lugar, permitiría observar las relaciones en espacios físicos, como también los sentidos atribuidos por los actores locales. Esta mutua relación se dinamiza aún más en la medida que los lugares de la ciudad se problematizan y relacionan a procesos de transformación (Lombard, 2015). No obstante, no se trata de una experiencia neutra, sino que emerge en relación a la agencia.

\section{Planteamiento metodológico}

El problema planteado en términos teóricos, plantea dos desafíos metodológicos. En primer lugar, atender las dinámicas socio-espaciales de intermediación en el estudio de ciudades intermedias implica adoptar una mirada interescalar. Esto permite identificar y articular diferentes interacciones socio-espaciales relevantes para el desarrollo de este tipo de ciudades, así como para comprender su articulación con otras escalas. En segundo lugar, el desafío de "lugarizar" estas ciudades y comprender sus particularidades, conlleva abordarlas empíricamente desde la diversidad de prácticas de actores claves que la habitan y producen socialmente. Es en este punto donde la complejidad de las ciudades intermedias logra situarse espacialmente y particularizarse socio-cultural, económica y ecológicamente. Esto conlleva no solo trascender acercamientos sectoriales y estáticos en los estudios urbano-territorial en pos de una examinación de problemas socio espaciales complejos, sino también comprender las prácticas y significados socio-espaciales asociadas a la intermediación (Salazar et al. 2017b).

El abordaje metodológico adoptado en este estudio es de carácter cualitativo, apoyándonos en el uso de datos socioeconómicos y espaciales. Esto posibilita no solo identificar variables y problemáticas complejas de intermediación sino también explorar sus prácticas y significados culturales constitutivos (Newing et al., 2010). Tal como muestra el Cuadro 1, en una primera fase, se han utilizado métodos cualitativos para identificar inductivamente cómo los actores locales entienden y operacionalizan las dimensiones teóricas de lugar de Massey (2012) en las tres ciudades en estudio. En una segunda fase, el análisis de estas dinámicas fue complementado con datos primarios y secundarios, con la finalidad de identificar tendencias generales en ámbitos morfológicos, económicos-productivos e interculturalidad. Las fuentes primarias empleadas incluyen los permisos de edificación para actividades comerciales de mayor (mall, supermercados, strip centers, obras mayores destinadas a comercio, etc.) y menor escala (almacenes minoristas, en general) que fueron otorgados por las direcciones de obras municipales de las comunas en estudio entre los años 2002 y 2012. Las fuentes secundarias incluyen datos de la encuesta CASEN 2013 y de los CENSOS poblacionales. A partir de esto, en una tercera fase, se exploraron y problematizaron las prácticas y significados constituyentes de las dinámicas de estudio a partir de 45 entrevistas realizadas en caminatas guiadas a través de la ciudad con sus habitantes (Anderson, 2004; Evans \& Jones, 2011). Finalmente, en una cuarta fase - y generando una metodología cíclica - se han redefinido y tensionado las dimensiones de análisis emergentes en función de los resultados obtenidos en las etapas dos y tres. 
Cuadro $\mathrm{N}^{\circ} 1$

Fases metodológicas

\begin{tabular}{|l|l|l|}
\hline FASE 1 & $\begin{array}{l}|c| \\
\text { Identificación de dinámicas emergentes } \\
\text { (inductivas) de intermediación }\end{array}$ & $\begin{array}{l}\text { Entrevistas semi estructuradas sedentarias } \\
\text { y caminadas por las ciudades / Análisis bi- } \\
\text { bliográfico }\end{array}$ \\
\hline FASE 2 & $\begin{array}{l}\text { Descripción complementaria de ten- } \\
\text { dencias generales en ámbitos económi- } \\
\text { co-productivos, morfológicos e intercul- } \\
\text { turales }\end{array}$ & $\begin{array}{l}\text { Sistematización de Permisos de edificación } \\
\text { 2002-2012; Caracterizaciones mediante } \\
\text { datos de Encuestas CASEN 2013 y CENSOS } \\
\text { 1982-2012, Chile }\end{array}$ \\
\hline FASE 3 & $\begin{array}{l}\text { Problematización y análisis de prácticas y } \\
\text { significados de intermediación en torno a a } \\
\text { dinámicas de análisis }\end{array}$ & $\begin{array}{l}\text { Entrevistas semi estructuradas sedentarias } \\
\text { y caminadas por las ciudades; procesos de } \\
\text { observación participante. }\end{array}$ \\
\hline FASE 4 & $\begin{array}{l}\text { Re-conceptualización de dinámicas emer- } \\
\text { gentes de intermediación }\end{array}$ & \multicolumn{2}{|c|}{} \\
\hline
\end{tabular}

Fuente: Elaboración propia.

Desde la perspectiva de los actores, en la fase 1 se identificaron tres dinámicas emergentes de intermediación correlacionadas con las tres categorías de lugar de Massey (2012). Dichas categorías ayudan a estructurar los resultados, a trascender generalizaciones y, por tanto, a entender la ciudad intermedia desde su condición de lugar:

1. Transformaciones morfológicas y relaciones sociales: la primera dimensión que surgió desde las entrevistas a actores fue la asociada a transformaciones en la morfología urbana derivadas de los procesos de intermediación de estas ciudades y la manera en que estas impactan en las relaciones sociales. Esta dimensión ha sido ampliamente trabajada en las ciudades intermedias latinoamericanas y chilenas (Arenas et al., 2009; Klaufus, 2010; Portes y Roberts, 2005; Canziani y Schejtman, 2014).

2. Relaciones de movilidad interescalar: tras una primera aproximación con los actores, estos enfatizan en el rol que tiene la movilidad como un aspecto que rompe las dicotomías urbano-rural y expresa formas de intermediación intensas entre la ciudad y su entorno más inmediato. Los trabajos de movilidad en Chile generalmente adoptan una perspectiva espacialista y no centran su interés en las prácticas de los actores (Salazar et al., 2017a; Jirón et al., 2016).

3. Relaciones interculturales: dado el contexto regional en el que se sitúa la investigación, surgió la temática indígena y la interculturalidad, como un asunto elemental asociado a las dinámicas de intermediación urbana. Si bien no hay trabajos específicos en la materia, autores como Aravena et al (2005) han dado cuenta de este proceso en las ciudades de la región de La Araucanía y del Biobío. 


\section{Hacia las especificidades del lugar: Caracterización socio-económica de intermediación entre las ciudades de estudio y su hinterland local}

Con el fin de presentar y caracterizar los casos de estudio, en este apartado se pone en valor cómo los procesos de intermediación socioeconómica de tres ciudades intermedias de la Región de La Araucanía con su hinterland local, contribuyen en la configuración de sus rasgos distintivos. Tanto sus rasgos comunes como aquellos con los que se diferencian las constituyen como casos claves para poner en perspectiva la importancia del lugar en los estudios de las ciudades intermedias.

Angol, Victoria y Villarrica (Figura $N^{\circ} 1$ ) son ciudades que cumplen importantes funciones de intermediación, articulando interacción con su hinterland inmediato y jugando un papel clave en los flujos socioeconómicos y de conmutación dentro del sistema urbano a nivel regional (Salazar et al., 2017a; Salazar et al., 2017b). Dada su localización en la región de La Araucanía, las tres son ciudades cruzadas por dinámicas de interculturalidad con una fuerte presencia en ellas del pueblo Mapuche. Estas características son claves para el desarrollo de estas ciudades y la comprensión de sus procesos de intermediación (Aravena et al., 2005; Salazar et al., 2017a). Ahora bien, aquí sostenemos que más allá de los rasgos comunes, Angol, Victoria y Villarrica poseen dinámicas socioeconómicas variadas, situación que resulta de las diferentes formas de intermediación que en ellas acontecen.

Figura $\mathrm{N}^{\circ} 1$

Región de la Araucanía y casos de estudios seleccionados

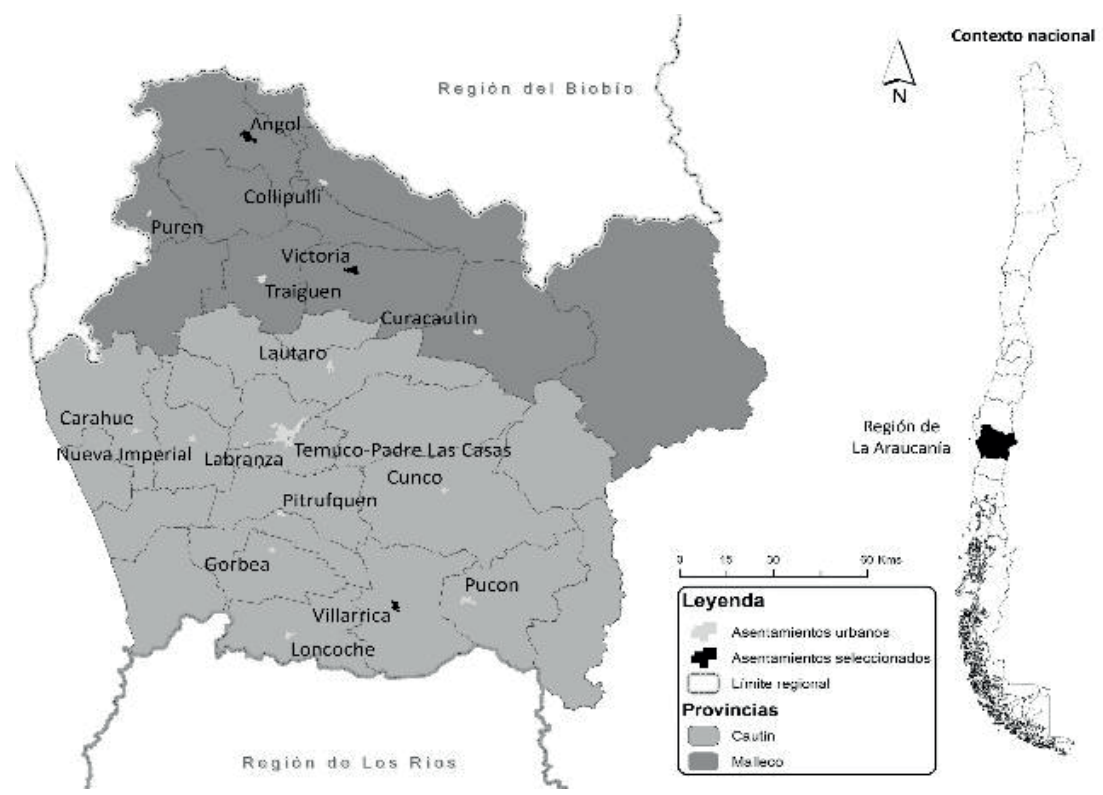

Fuente: Elaboración propia. 
Las tres ciudades han tomado funcionalidades diferentes en el sistema regional, lo que se puede observar en la Figura $\mathrm{N}^{\circ} 2$. Si bien, se podría afirmar que estas tres ciudades se encuentran asociadas a actividades primarias, en la práctica tienen elementos divergentes. Villarrica presenta una composición de empleo de mayor diversificación, en donde la rama industrial y construcción marcan diferencia, mientras que en Angol y Victoria la prevalencia del sector silvoagropecuario es notoria. En ese sentido, Angol y Victoria podrían asociarse a las ciudades de base agrícola planteadas por Canales y Canales (2013) y, Portes y Roberts (2005); sin embargo, las tendencias demográficas de Victoria plantean matices. Lejos de tener un auge demográfico reciente, la comuna ha mostrado estancamiento, e inclusive, decrecimiento en el último periodo censal (Cuadro $\mathrm{N}^{\circ} 2$ ). Pese a su importante base silvoagropecuaria, Victoria no se vio potenciada por el auge exportador en Chile y América Latina. Por su parte, pese a no tener una base primaria como tal, Villarrica sí ha aumentado su población de forma sostenida desde 1982, especialmente por la intensificación del turismo (Zunino y Hidalgo, 2010). De este modo, los datos cuantitativos por sí solos ya van dando cuenta de las importantes diferencias entre las tres ciudades, pese a que tienen márgenes demográficos similares.

Figura $\mathrm{N}^{\circ} 2$

Porcentaje de trabajadores por rama productiva en las comunas de Angol, Victoria y Villarrica

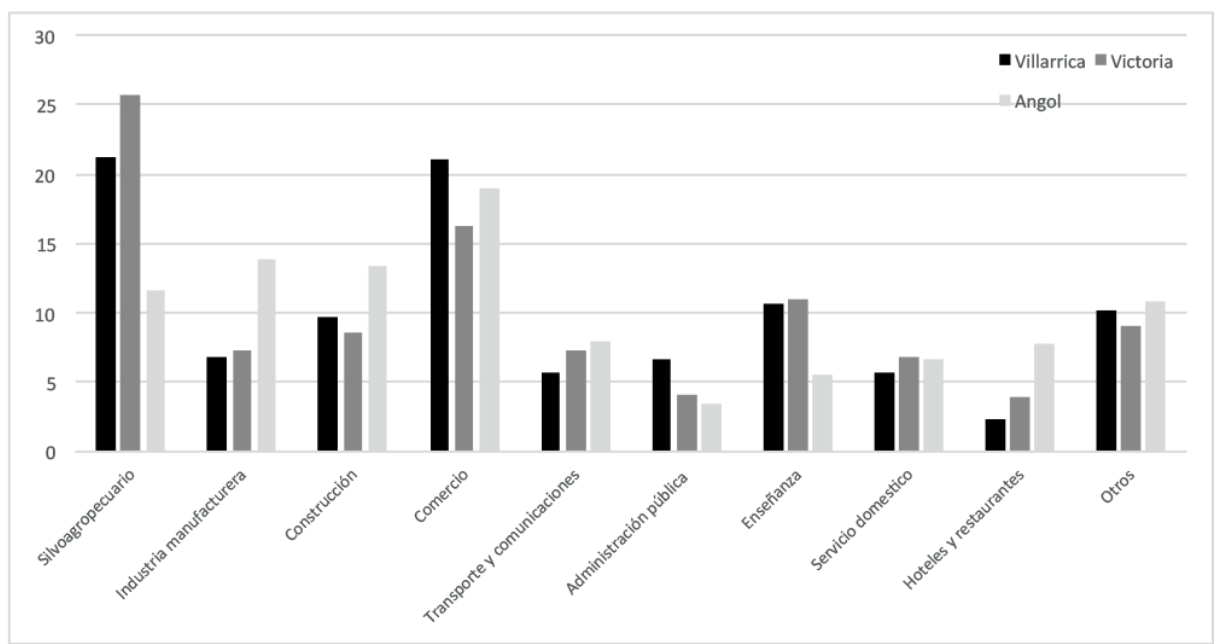

Fuente: Elaboración propia basada en CASEN 2013.

Cuadro $\mathrm{N}^{\circ} 2$

Tendencia demográfica de la población a nivel país, regional y comunas de Villarrica, Angol y Victoria entre los años 1960 y 2012

\begin{tabular}{|l|r|r|r|r|r|r|}
\hline & \multicolumn{1}{|c|}{1960} & \multicolumn{1}{c|}{1970} & \multicolumn{1}{c|}{1982} & \multicolumn{1}{c|}{1992} & \multicolumn{1}{c|}{2002} & \multicolumn{1}{c|}{2012} \\
\hline Villarrica & 24.366 & 28.177 & 32.973 & 35.867 & 45.531 & 51.511 \\
\hline Angol & 30.623 & 35.053 & 39.599 & 46.226 & 48.996 & 50.821 \\
\hline Victoria & 26.874 & 28.356 & 31.530 & 32.979 & 33.501 & 32.890 \\
\hline
\end{tabular}

Fuente: Elaboración propia basado en datos del Instituto Nacional de Estadísticas (varios años). 
La base productiva de Villarrica incide en que sus dinámicas de intermediación se encuentran estrechamente relacionadas con sus alrededores. Las transformaciones que ha experimentado la ciudad en las últimas décadas han sido causadas por la atracción de personas y capitales hacia los hitos naturales de la zona, convirtiéndola en uno de los principales destinos turísticos del sur de Chile. La actividad turística ha potenciado la economía local diversificando el mercado de trabajo de la ciudad. Por lo tanto, las dinámicas socio-espaciales de Villarrica en la actividad deben ser entendidas como el resultado de una configuración socioeconómica basada en una estrecha vinculación entre la ciudad y su entorno inmediato.

La intermediación en Victoria está sustentada en la entrega de servicios fundamentalmente al entorno rural y comunas aledañas (por ejemplo, Collipulli, Traiguén, Lumaco, Galvarino). Sin embargo, el tipo de intermediación ejercida por la ciudad se ha ido transformando en el tiempo en función de cambios productivos y su relación con Temuco, capital regional. De ese modo, si bien la intermediación es asociada a la función de servicios, esta sería problematizada entendiendo que dicho rol urbano ha disminuido junto al decrecimiento de las actividades productivas. La intermediación en Victoria se encuentra lejos de la que se podría imaginar para comunas con importante base primaria, pese a lo relevante de este sector en términos de mercado de trabajo. Esto se puede entender en buena medida porque el sector silvoagropecuario emplea prácticamente a la totalidad de la población rural de la comuna, pero que no definen para los habitantes de Victoria que su ciudad sea fundamentalmente agrícola.

De los tres casos abordados, Angol es sin duda el que mejor representa una economía basada en actividades primarias. La intermediación de la ciudad de Angol está dada principalmente por la influencia que tiene la producción frutícola en el carácter de la ciudad, siendo el empleo principal de gran parte de la población urbana (21,15\%), históricamente relacionado al fundo "El Vergel". La población de la comuna de Angol reside fundamentalmente en la zona urbana, y ese es el sitio de proveniencia de la mayor parte de los trabajadores silvoagropecuarios. Si bien Angol es lo más similar a una "agropolis" dentro de las ciudades abordadas, en ningún caso esto significa que no cuente con una comprensión temporal específica generada por la forma de sus interacciones socio-espaciales. En efecto, es esa condición la que sirve como contrapunto para ilustrar la heterogeneidad de estas ciudades intermedias.

En definitiva, aunque los casos de estudio aquí presentados pueden ser catalogados como ciudades intermedias, en términos socioeconómicos presentan dinámicas que difieren significativamente entre ellos. Por un lado, mientras en términos económicos Angol podría ser catalogada como una "agrópolis" (Canales \& Canales, 2013), Victoria en cambio debiera ser considerada una "serviciopolis" y Villarrica una "turismopolis". La heterogeneidad en las características socioeconómicas de estas ciudades son un indicio de la importancia que tienen avanzar hacia la "lugarización" de la ciudad intermedia.

\section{Transformaciones morfológicas y relaciones sociales, movilidad interescalar e interculturalidad de Villarrica, Angol y Victoria}

Cada una de las dimensiones abordadas se orienta a analizar las dinámicas de intermediación de los casos de estudio y atender las preguntas de investigación planteadas. A partir del relato y 
las prácticas espaciales de los actores, se da cuenta de la necesidad de distinguir la especificidad de cada una de estas ciudades. Esto no solo nos lleva a entender las condiciones de lugar de cada ciudad, sino que también a cuestionar los universalismos que han sido generados hasta ahora por la discusión sobre las ciudades intermedias.

\section{La intermediación y las transformaciones morfológicas: ciudades mo- no-centrales ¿El paraíso para la integración?}

Hay cierto consenso en que las características morfológicas de las ciudades intermedias las convierten en lugares menos segregados. Una de las condiciones esenciales para ello es la existencia de un solo centro, situación que entrega la oportunidad de que todos sus habitantes puedan confluir en un mismo lugar, independiente las características económicas o étnicas que ellos posean. Inspirados en Massey (2012) aquí argumentamos que aquella generalización, que resulta de una relación mecánica entre lo espacial y social, no es tan simple. En efecto, las prácticas de los actores en los casos de estudio ilustran que la monocentralidad de las ciudades intermedias no necesariamente se traduce en menos segregación y, por tanto, mayor integración social.

Los cambios morfológicos de las ciudades intermedias han sido concomitantes con sus dinámicas demográficas y sus dinámicas de intermediación. Villarrica, que tiene el crecimiento poblacional más importante de las ciudades en estudio, presenta las transformaciones más significativas. La comoditización de la naturaleza que rodea a la ciudad ha impulsado el arribo de importantes inversiones turísticas como hoteles de lujo, condominios cerrados, servicios turísticos especializados y también nueva población que ha arribado buscando oportunidades de inversión-trabajo o un nuevo estilo de vida. En este sentido, la ciudad ha incrementado sus redes de intermediación en todas las escalas territoriales. En cambio, Angol y Victoria han cambiado de manera más lenta. Si bien ambas ciudades han experimentado crecimiento poblacional, este ha sido reducido. En ambas ciudades las dinámicas de intermediación parecen estar dirigida hacia zonas y escalas territoriales con muy poco dinamismo socioeconómico, lo que le ha hecho perder protagonismo en el contexto regional y ha marcado el ritmo de sus transformaciones urbanas. Aunque ello no niega los cambios. Tanto Angol como Victoria han crecido horizontalmente, lo que se debe mayoritariamente a la construcción de conjuntos de vivienda social en las zonas periféricas y a la renovación que han experimentado algunos de sus barrios luego del terremoto que afectó la zona en 2010. Así, las dinámicas poblacionales y de intermediación específicas de Villarrica, Victoria y Angol, hacen que los cambios morfológicos en estas tres ciudades intermedias muestren ritmos propios.

Las Figuras $\mathrm{N}^{\circ} 3, \mathrm{~N}^{\circ} 4$ y $\mathrm{N}^{\circ} 5$ dan cuenta de los permisos de edificación comerciales que se han entregado entre 2002 y 2012 en las tres ciudades intermedias en estudio. Dichas figuras muestran que, aunque con ritmos de transformación propios y con algún grado de dispersión funcional (especialmente en Villarrica y Angol), las tres ciudades poseen la mayor parte de su actividad comercial concentradas espacialmente, esto significa que son ciudades monocentrales. Por lo tanto, más allá de que tengan ritmos de transformación propios, son ciudades que como rasgo específico solo tiene un centro. Se confirma así lo que plantea la literatura respecto a una de las características esenciales de las ciudades intermedias. No obstante, cabe preguntarse ¿cómo esta característica morfológica se conecta con el tipo de relaciones sociales que los habitantes de estas ciudades despliegan? Lo que implica interrogarse si efectivamente esta característica espacial tiene la capacidad de producir integración social en las ciudades intermedias. 
Según la percepción de algunos de los actores locales, el centro de estas ciudades efectivamente se constituye como un lugar de "encuentro" social. Como todos los servicios urbanos se concentran en el centro o cercano a este, los habitantes concurren hasta él de forma periódica. Sin embargo, ello no significa que automáticamente se estuvieran produciendo procesos de integración social. Lo que ocurre es que se desarrollan prácticas de distinción y micro segregación de carácter más sutil que obstaculizan el encuentro entre personas más allá de sus diferencias socioeconómicas o étnicas. El comercio, es un aspecto a través del cual se observa esta condición.

En Angol los actores sociales reconocen una distinción importante entre los comercios ubicados alrededor del terminal rural y los localizados entre la plaza de armas y la Municipalidad. Alrededor del terminal rural es posible encontrar "La Farco" (centro comercial popular) y abundante comercio callejero que ofrece productos que se cosechan en la escala inmediata a Angol. En tanto, alrededor de la municipalidad, el terremoto del 2010 derrumbó una cantidad importante de viviendas y negocios antiguos que hoy están siendo reemplazados por cadenas comerciales de carácter nacional. Aunque ambos lugares se encuentran en el centro y muy cercanos, los perfiles socioeconómicos de quienes los emplean son diferentes. Mientras alrededor del terminal es común encontrar a personas de edad avanzada, que habitan en zonas rurales y sin una situación económica acomodada, en la zona en proceso de renovación, las familias poseen una situación económica más consolidada, con acceso al crédito y un marcado estilo de vida urbano.

En Villarrica la distinción social parece adquirir un carácter más específico. En esta ciudad es usual encontrar en las calles comerciantes que ofrecen frutas y verduras frescas provenientes de huertas cercanas. Además, pequeñas ferias, distribuidas por todo el centro, donde se ofrecen artesanías en madera, telares, etc. Debido a que estos lugares reflejan lo propio de Villarrica, son frecuentados tanto por habitantes locales como por turistas, más allá de las diferencias de ingreso o étnicas que estos puedan poseer. Sin embargo, la actividad turística también ha impulsado el desarrollo algunos negocios dirigidos a un público más específico. Entre ellos el negocio de la comida. En los últimos años se ha observado el arribo de restaurantes de alta gama, especialmente en la zona cercana a la costanera de la ciudad. La mezcla de comida gourmet y vista al lago y al volcán es un privilegio que no está dirigido ni a todos los habitantes de Villarrica ni a todos los turistas que arriban hasta ella, sino que mayoritariamente a personas con un capital económico y cultural alto.

Entonces, aunque el centro de estas ciudades intermedias se constituye como un punto de encuentro de la diversidad, ello no necesariamente implica que su condición de monocentralidad produzca una intensificación de las relaciones sociales, hay barreras que operan a nivel micro-social o micro-espacial que las tienden a obstaculizar. Como ilustra el caso de Angol, una de esas barreras la constituye el tipo de comercio que se ubica en el lugar, en tanto que como muestra Villarrica, también la comida y la vista pueden operar como mecanismos de distinción.

En último término, estos resultados muestran una contradicción entre los fenómenos espaciales y sociales que se opone a la visión dominante en los estudios sobre las ciudades intermedias. La monocentralidad de estas ciudades no implicaría menor segregación. Se problematiza así la relación entre lo espacial y social mostrando que esta no es para nada mecánica, sino que adquiere particularidades que varían caso a caso. Dichas particularidades y contradicciones no niegan la 
unicidad de este tipo de ciudades, sino que se constituyen en aspectos claves en la conformación de su naturaleza o la esencia de las ciudades intermedias.

Figura $\mathrm{N}^{\circ} 3$

Hitos y permisos de edificación comerciales en Villarrica

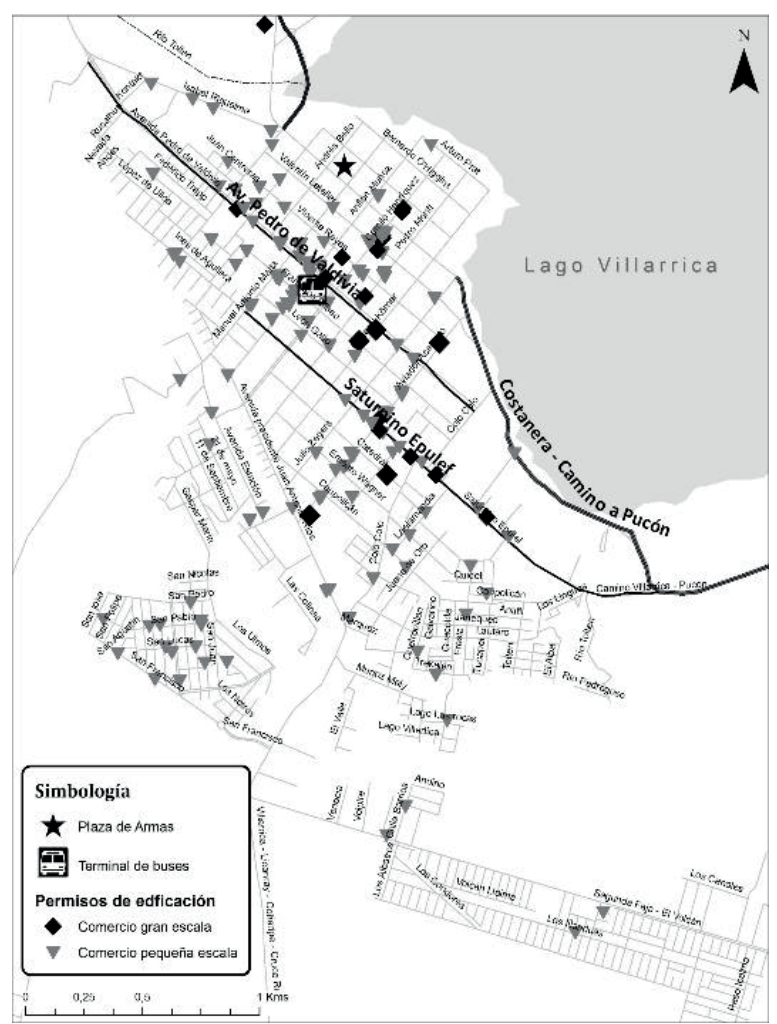

Fuente: Elaboración propia.

Figura $\mathrm{N}^{\circ} 4$

Hitos y permisos de edificación comerciales en Victoria

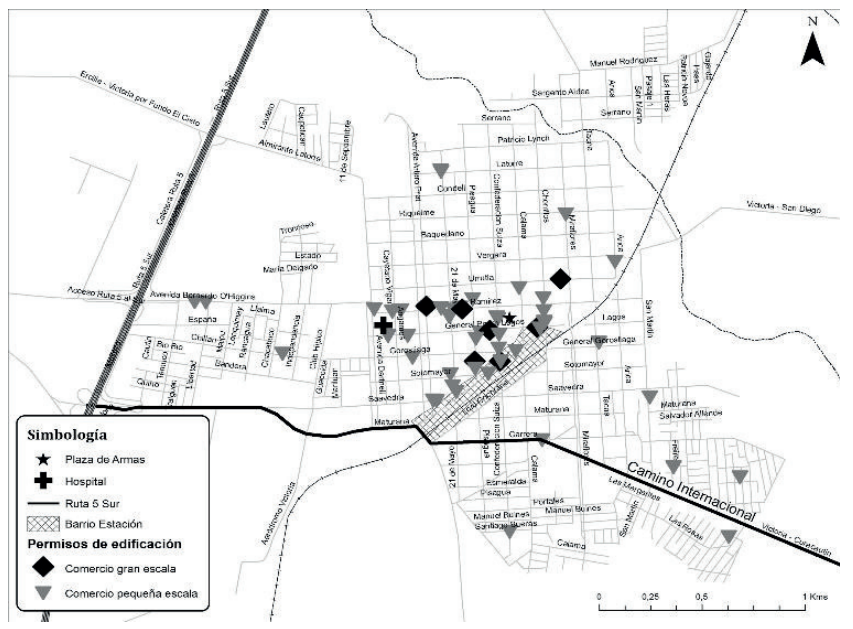

Fuente: Elaboración propia. 
Figura $\mathrm{N}^{\circ} 5$

Hitos y permisos de edificación comerciales en Angol

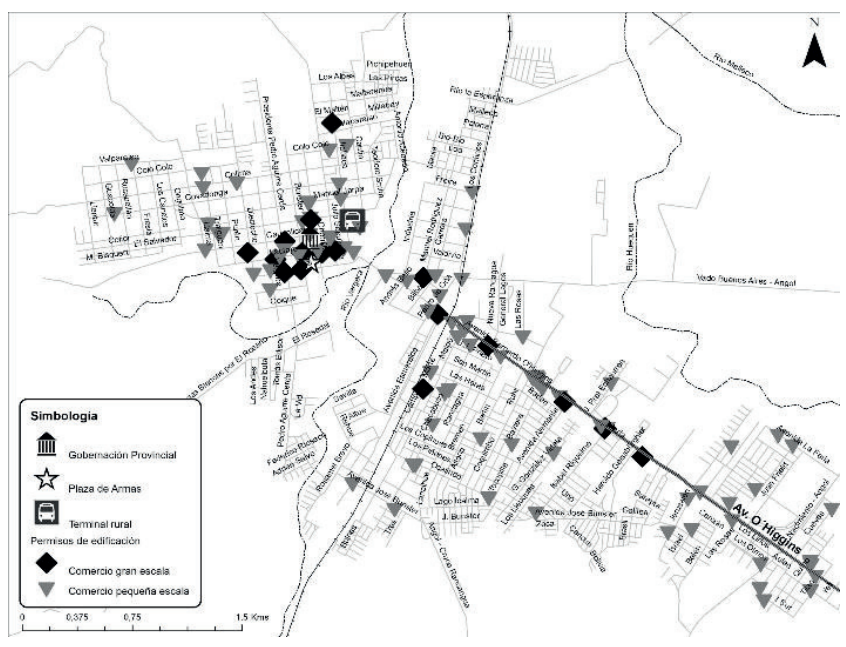

Fuente: Elaboración propia.

\section{Desde la intermediación con las escalas predefinidas a fronteras emergentes desde la experiencia del lugar}

En términos conceptuales, la literatura ha enfatizado que los asentamientos urbanos intermedios establecen relaciones escalares que son fundamentales para su desarrollo. Concretamente se han propuesto tres escalas de intermediación: entre la ciudad y su hinterland local; entre la ciudad y otros sistemas urbanos metropolitanos; y entre la ciudad y dinámicas económicas globales (Bellet y Beltrao 2009). Estas escalas han sido importantes para dejar atrás perspectivas estáticas y demográficas propias del concepto de "ciudad media". Sin embargo, si bien la literatura ha adoptado una mirada sistémica en la cual se vinculan distintas unidades escalares, éstas aún aparecen como unidades en sí mismas, definidas a priori, lo que dificulta atender -o dejando en un segundo plano- las prácticas socio-espaciales que hay detrás. A la luz de Massey (2012), argumentamos que esto representa una generalización que no ayuda del todo avanzar hacia un entendimiento relacional de este tipo de ciudades. En ese sentido, la relación entre la ciudad y sus diversas escalas de intermediación tiende a ser entendida como instrumento para el estudio de estados espaciales mayores (como sistemas interurbanos, metropolitanos o urbano-rural) y no como -en línea con Massey (2012)- procesos socio-espaciales que -de facto- constituyen a la ciudad intermedia dinámicamente. El riesgo que posee estudiar la relación generalizada entre centros urbanos y sus escalas de intermediación está en fijar conceptualmente fronteras espaciales a priori, que limitan la comprensión profunda de la condición de lugar. Las fronteras del lugar como ejercicio operativo, tal como señala Massey, amenazan con establecer categorías que invisibilizan la complejidad con la que se van tejiendo las escalas desde la experiencia del lugar. Por el contrario, aquí postulamos que es imperante avanzar más en una conceptualización "relacional" de la ciudad intermedia y que eso descansa fundamentalmente en atender las prácticas socio-espaciales que subyacen a estas distintas escalas de intermediación y a su condición de lugar. 
Al caminar con los entrevistados fue posible observar cómo las escalas planteadas en la literatura se entrelazan en la experiencia del lugar. Las estaciones de buses inter rural y el comercio informal articulado a su alrededor en los tres casos de estudio, ilustran claramente cómo las dinámicas urbanas están imbricadas en un complejo flujo de movilidades que conectan lugares y experiencias. En las paredes de las estaciones y en la parte delantera de los buses, hay letreros que mencionan nombres de localidades rurales a los cuales dirigen su destino. Su flujo genera un ritmo constante de interconexión entre la ciudad y el hinterland. Actores asociados al comercio informal urbano lo viven día a día en sus puestos de venta de productos rurales afuera de los supermercados de las grandes cadenas de comercio. Un número significativo de población vende productos recolectados del campo que dependen de la estacionalidad del territorio. La ciudad se encuentra profundamente interconectada al territorio circundante por medio de estas prácticas de comercio y movilidad.

Esta interconexión de las escalas en la experiencia del lugar es vivida desde la experiencia corporal (Salazar et al., 2017a; Salazar et al., 2017b; Ricketts et al., 2008; Sheller \& Urry, 2006). Recorriendo la segunda faja de Villarrica (sector de viviendas sociales), la intermediación se hace presente en las atmósferas y olores que evidencian un entendimiento corporal de la simultaneidad de escalas. Desde la calle que conecta estos sectores con los centros de la ciudad, se destacan olores de fecas de animal asociados a la producción ganadera del hinterland urbano. Este fenómeno es relatado por vecinos del sector aumenta en los días de verano. Las escalas son vividas con el cuerpo desde la experiencia del lugar.

El hecho que la intermediación se constituya dinámicamente por procesos de movilidad -entrelazando diversas escalas- supone también la emergencia de fronteras. Ahora bien, estas fronteras no tienen que ver con aquellas que se puedan identificar desde escalas de intermediación predefinidas (como por ejemplo la urbana-rural o lo local-global) sino que son inherentes a la condición de lugar en donde confluyen fuerzas y prácticas desde diversas escalas.

Volviendo a la venta informal, al moverse a vender a la ciudad, los límites que se viven son de una espacialidad más compleja que la escala ciudad-hinterland. En su experiencia de movilidad, los límites están dados por los accesos a la venta y la instauración de barreras comerciales bajo la distinción de trabajo formal e informal, según el pago de impuesto y el arriendo de espacio para vender. De este modo, las fronteras del lugar se expresan en las tensiones inmanentes al lugar en términos de acceso, alterando la experiencia de movilidad relacional entre escalas.

Esto también se expresa en la intermediación con dinámicas globales extractivitas, como la masificación de la industria forestal en los alrededores de Angol. Varios actores comentan que la expansión de esta industria en la última década no solo ha reemplazado grandes extensiones de suelos agrícolas y de bosque nativo, sino también ha transformado la experiencia de movilidad entre la ciudad y su hinterland. Las experiencias de una ciudad históricamente compenetrada con diversas prácticas agrícolas en sus territorios circundantes van quedando como retazos de un paisaje dominado cada vez más por bosques de pino. Tal como lo expresó un funcionario de la Dirección de Planificación de Angol, emerge una ciudad rodeada de un "desierto verde", transformando la interconexión entre las escalas. A su vez, en Villarrica los vendedores de productos forestales no madereros ilustran las transformaciones del entorno ambiental desde las pérdidas de hongos para vender en la ciudad. Hongos asociados al roble pellín (Nothofagus obliqua) tales 
como el gargal (Grifola gargal), comúnmente consumidos y valorados por la población local, ya no son encontrados, ni se encuentran disponibles. Esta pérdida se encuentra asociada a la expansión de la industria forestal y la explotación del bosque nativo. En la experiencia de pérdida de este tipo de productos nativos se materializa la transformación en la interconexión entre sectores rurales y la ciudad. De tal modo, en la experiencia del lugar se expresan dinámicas globales y regionales, que a través de políticas de incentivo forestal exótico y falta protección del bosque nativo, han alterado la condición de lugar.

\section{Identidad y ciudad intermedia: Entre las prácticas de intermediación intercultural y su invisibilización}

La literatura de ciudad intermedia ha asociado a estos asentamientos urbanos con la posibilidad de una planificación local más pertinente al presentar identidades locales homogéneas e identificables. Esto es evidente en las ciudades de estudio y las dinámicas asociadas a la interculturalidad.

La Araucanía es la segunda región con mayor población rural en Chile, con un 32,3\%, y la que cuenta con mayor proporción de población indígena del país, con un 32\% (CASEN 2013). Esto conlleva dos alcances importantes para la comprensión de la condición de lugar de las ciudades de estudio. Por un lado, la interculturalidad se expresa en la relación entre escalas de intermediación, específicamente en dinámicas de movilidad entre la ciudad y su hinterland local. Por otro lado, estas dinámicas de movilidad intercultural están sujetas a fuertes proceso de tensión e invisibilización en el espacio, lo que nos lleva a cuestionar universalismos sobre identidades estáticas y homogéneas asociadas a este tipo de ciudades. Estas tensiones e invisibilizaciones se expresan de forma diversa en los casos estudiados.

Las características demográficas de Victoria en términos de ruralidad y población mapuche son muy cercanas a la media regional. Si bien la población mapuche en la comuna de Victoria representa cerca de un tercio del total comunal, la mayor parte de ella reside en zonas rurales, representando el $71,2 \%$ de la población rural (CASEN, 2013). Por consiguiente, al abordar la intermediación entre la ciudad y su entorno inmediato, se debe entender que en Victoria la mayor parte de la zona rural pertenece a la etnia Mapuche. Esto incide en el paisaje urbano de dos modos. Primero, la ciudad de Victoria está marcada por dinámicas del mundo rural que se expresan en prácticas urbanas cotidianas, principalmente por procesos de conmutación diaria. En las veredas y calles del barrio estación - particularmente en las inmediaciones del paradero de locomoción interurbanos - población mapuche ofrece productos rurales, y de ese modo es que se materializa la intermediación en el paisaje urbano. Segundo, la ciudad se ha convertido en un nodo urbano-territorial para la facilitación del sector silvoagropecuario en la comuna, el cual representa un $25,71 \%$ del total del empleo comunal. Un $71,5 \%$ de la población empleada en dicho sector es mapuche, y ya sea de forma independiente o proletarizada, esto se torna es un aspecto distintivo de las prácticas de intermediación intercultural en la ciudad de Victoria. Desde este punto de vista, la intermediación pasa principalmente por la relación de uso del espacio urbano de parte de la población ubicada en espacios rurales, principalmente de origen mapuche. Sin embargo, los actores entrevistados coinciden en que los lugares donde se dan estas prácticas de interacción intercultural, son espacios marginalizados de la ciudad y, en consecuencia, la interacción con el entorno inmediato es invisibilizada. Esta marginalización queda mayormente ilustrada en los casos de Villarrica y Angol. 
Si bien la ciudad de Villarrica también presenta fuertes dinámicas de intercambio intercultural, este es causalmente distinto al caso de Victoria, debido a: 1) una reconfiguración de relaciones interculturales producto de las amenidades de la zona que han gatillado un enérgico auge del sector del turismo en los últimos años; y 2) un fuerte proceso de migración campo-ciudad en las últimas décadas que ha significado, entre otros fenómenos territoriales, que actualmente el $53,4 \%$ de la población mapuche de la comuna viva en la ciudad, y que esta represente un $21,8 \%$ de la población urbana (CASEN 2013). Esto conlleva dos dinámicas de intermediación que complejizan bastante el fenómeno de la interculturalidad en Villarrica. Primero, al igual que en Victoria, la ciudad de Villarrica sigue siendo un nodo de intercambio de productos y servicios rurales que se ofrecen en puestos de venta, ferias y comercios informales enfocados principalmente al turismo. A su vez, es un centro de abastecimiento vital para numerosas comunidades indígenas y no indígenas de una gran parte de la cuenca del río Toltén. En ese sentido, tal como lo expresan múltiples actores locales, Villarrica funciona como el nodo central de un territorio funcional de connotación intercultural. Segundo, la alta presencia de población mapuche urbana abre también nuevas dinámicas y prácticas interculturales. Sin embargo, a diferencia de fenómenos de mapuche urbanos en Santiago de Chile, aquí la proximidad a los territorios ancestrales ha generado que la población mapuche urbana de Villarrica no haya perdido una cercanía cotidiana con los sectores rurales y por ende identitaria - en términos de redes y lazos familiares -, de tal modo que "el acceso a la identidad de origen está siempre presente, al alcance de la mano, a una o dos horas de viaje en bus" (Aravena et al., 2005: 129). De este modo, la presencia ancestral indígena, pasando por los procesos de ocupación del Estado Chileno en la zona y las recientes transformaciones del territorio planteadas en este estudio, configuran un complejo y diverso escenario de intermediación intercultural. A pesar de aquello, diversos actores de la ciudad de Villarrica relatan una fuerte invisibilización de lo Mapuche en la ciudad, predominando una visión folklorizada de la cultura enfocada hacia el turismo, y negando las prácticas interculturales relacionadas a la intermediación, tal como lo proponen dirigentes de organizaciones urbanas mapuche entrevistados. Este caso muestra como en la condición de lugar de la ciudad, antes que una expresión de identidad, se expresan conflictos y procesos históricos de negación espacial, invisibilizando el rol de la intermediación.

Por su parte, Angol tiene una dinámica de intermediación intercultural diametralmente opuesta a los casos de Villarrica y Victoria. Pese a estar situada en el territorio histórico mapuche, la comuna cuenta con una muy baja cantidad de población perteneciente a dicho grupo indígena $(9,2 \%)$ y solo un $5 \%$ de su población urbana se autodenomina como perteneciente a la etnia Mapuche. En cierto modo, se debe considerar que Angol no solo fue fundada siete veces hasta que la ocupación de La Araucanía se hizo efectiva con una fuerte expulsión indígena, sino también que debido a su condición de micro-clima y de tierras con buena vocación productiva se tendió a desplazar a la población mapuche hacia sectores menos productivos. Desde entonces, las transformaciones urbanas han estado estrechamente vinculadas a una ruralidad abocada al sector agro exportador. Sin embargo, desde la perspectiva de varios actores entrevistados, esto ha sucedido mano a mano con una invisibilización territorial de la cultura mapuche y a un deterioro ecológico en la zona. En este sentido, en la condición de lugar es fundamental considerar la expresión de fuerzas históricas que se plasman en la propia condición y las prácticas cotidianas. Debido al desplazamiento continuo de la cultura mapuche de la ciudad, los procesos de reivindicación indígena en esta zona se han entendido como ajenos a lo urbano, como un fenómeno meramente rural. Es en las comunas vecinas de Angol, en donde se ha configurado la "zona roja" del conflicto chileno-mapuche. Así, la ciudad se encuentra lejos de ser centro de manifestación cívica (Salazar et al. 2017a). 
A la luz de estos tres casos, la experiencia de lugar ilustra que las identidades asociadas a la ciudad intermedia no son fijas, ni estables. Toman forma en la interconexión con los sectores rurales por medio de constantes prácticas de movilidad intercultural. Ahora bien, a pesar de estas dinámicas de movilidad intercultural, los casos de estudio reflejan complejos procesos de invisibilización. Por lo tanto, las dinámicas de homogeneización identitaria no son inherente a el concepto de ciudad intermedia, sino que corresponden a relaciones de poder que van fijando identidades en el tiempo. En consecuencia, el lugar nos ayuda a entender la interculturalidad como un proceso en constante tensión en el espacio y comprender la ciudad intermedia desde su heterogeneidad.

\section{Conclusión}

En la última década, la literatura de ciudad intermedia -basada, principalmente, en metodologías espaciales y cuantitativas- ha avanzado en determinar escalas de intermediación que integran funcionalmente a la ciudad con fenómenos escalares mayores. Sin duda esto ha permitido trascender perspectivas antecesoras de carácter estático y demográficas respecto de este tipo de asentamientos. Ahora bien, en este artículo hemos argumentado que este avance aún posee algunas dificultades conceptuales y metodológicas para abordar la noción de intermediación, en tanto ha tendido a desarrollar descripciones y fundamentaciones de tendencia universalista que no permiten examinar en profundidad las complejidades socio-espaciales de este tipo de ciudades. En ese sentido, vincular la conceptualización de intermediación con la noción de lugar nos permite llevar el estudio de estas ciudades a una dimensión más profunda, hasta ahora poco explorada en la literatura, como también desarrollar una perspectiva relacional para su entendimiento. Respecto de esto último, la noción de lugar nos permite ilustrar prácticas y experiencias socio-espaciales constitutiva de la ciudad y sus respectivas dinámicas de intermediación. En otras palabras, entender la ciudad intermedia como lugar conlleva -tanto teórica como metodológicamente- poner en el centro a las relaciones socio-espaciales como constructos dinámicos de intermediación escalar.

A la luz de este trabajo es posible destacar cómo en la aproximación del lugar emergen tensiones para su comprensión específica. Se trata de un movimiento dialéctico entre tensiones particulares y la experiencia del lugar. El lugar va generando tensiones y las tensiones van creando lugar. El aporte de este artículo se encuentra en examinar cómo, particularmente, opera esta dialéctica en las dinámicas de intermediación. Específicamente los resultados de los casos de estudio ilustran la existencia de tensiones socio-espaciales, de movilidad interescalar y de relaciones interculturales que van dinámicamente construyendo la dialéctica del lugar. Las tensiones operan de una manera inmanente a la experiencia del lugar, tal como lo expresan los procesos descritos en los resultados. Como inmanente nos referimos a que dichas tensiones se encuentran ancladas en la experiencia de los lugares y las prácticas socio-espaciales y, por tanto, para sus análisis deben ser consideradas como indisolubles. Es necesario recalcar que bajo este enfoque no se pierde la unicidad del lugar, tal como lo plantea Massey. De este modo, al enfocarnos en el lugar - esto es, en las prácticas socio-espaciales y sus tensiones emergentes - es posible expandir la comprensión del enfoque de intermediación y el de ciudades intermedias.

Enfocarse en la intermediación por medio del enfoque de lugar nos ha permitido identificar y explicar las tensiones y desafíos específicos de cada ciudad. Para los casos y variables particula- 
res que hemos estudiado emergen tres puntos claves en materia de planificación: 1) impulsar la integración social a partir de la monocentralidad que es característica en estas ciudades; 2) promover un entendimiento interescalar de la movilidad constitutiva de estos asentamientos con el fin de integrar territorios vinculados relacionalmente; y 3) Asumir la heterogeneidad y las tensiones inherentes al carácter de estas ciudades como motor de una mayor visibilización intercultural. Estas especificidades, en último término, son claves para el desarrollo de una planificación más apropiada. Ilustra la importancia metodológica de promover el estudio de la ciudad intermedia y su respectiva planificación desde las experiencias del lugar. Con todo, es importante seguir expandiendo esta propuesta en metodologías mixtas que acoplen integralmente la ciudad intermedia a las dinámicas del lugar del que son parte.

\section{Referencias bibliográficas}

ANDERSON, J. Talking whilst walking: a geographical archaeology of knowledge. Area, 2004, Vol. $36, N^{\circ} 3$, p. 254-261.

ARAVENA, A., N. GISSI Y G. TOLEDO. Los mapuches más allá y más acá de la frontera: identidad étnica en las ciudades de Concepción y Temuco. Sociedad Hoy No 8 p.117-132

ARENAS, F.; GONZÁLES, D. y ALIAGA, G. La conurbación Rancagua-Machalí: transformaciones socioespaciales de una ciudad media. En: HIDALGO, R.; DE MATTOS, C. y ARENAS, F. Chile: Del país urbano al país metropolitano. Santiago de Chile: GEOlibros-Colección EURE-libros, 2009, p. 347358.

BELLET, C. y BELTRAO, M. (editors). Las ciudades medias o intermedias en un mundo globalizado Lleida: Edicions de la Universitat de Lleida, 2009.

BELLET, C. y LLOP, J. Miradas a otros espacios urbanos: las ciudades intermedias. Scripta Nova. Revista Electrónica de Geografía y Ciencias Sociales, 2004, Vol. VIII, № 165. Disponible en Internet: http://www.ub.edu/geocrit/sn/sn-165.htm

BERDEGUÉ, J.A.; CARRIAZO, F.; JARA, B.; MODREGO, F. y SOLOAGA, I. Cities, Territories, and Inclusive Growth: Unraveling Urban-Rural Linkages in Chile, Colombia, and Mexico. World Development, 2015, Vol. 73, p. 56-71.

BERDEGUÉ, J.A.; JARA, B.; FUENTEALBA, J.; TOHÁ, J.; MONDREGO, F.; SCHEJTMAN, A. y BRO, N. Territorios funcionales en Chile. Santiago de Chile: Documento de trabajo, RIMISP. Programa Dinámicas Territoriales Rurales, 2011, № 102.

BEREZIN, A. Chaitén: una historia en el lugar. Magallania, 2015, Vol 43, № 3, p. 91-106.

BOLAY, J.-C. \& RABINOVICH, A. Intermediate cities in Latin America risk and opportunities of coherent urban development. Cities, 2004, Vol. 21, N 5, p. 407-421.

BORSDORF, A.; SÁNCHEZ, R. y MARCHANT, C. Aprendiendo de los errores. La necesidad de cambios a la política nacional de vivienda en ciudades intermedias chilenas. Scripta Nova. Revista 
Electrónica de Geografía y Ciencias Sociales, 2008, Vol. XII, № 270. Disponible en Internet: http:// www.ub.edu/geocrit/-xcol/166.htm

BORSDORF, A.; MARCHANT, C. \& SÁNCHEZ, R. Changes in urbanization processes. The intermediate cities in the Chilean urban system. In: RIVERA SALAZAR, D. (editor). Chile: Environmental, Political and Social Issues. Hauppauge, N.Y.: Nova Publishers, 2012, p. 159-173.

CANALES, A.I. y CANALES, M. De la metropolización a las agrópolis: El nuevo poblamiento urbano en el Chile actual. Polis (Santiago), 2013, Vol. 12, N³4, p. 31-56.

CANZIANI, J. y SCHEJTMAN, A. Ciudades intermedias y desarrollo territorial. Lima: RIMISP, 2013.

DA SILVA, V. O bairro na pequena cidada: para alem da identidade, o conflicto. Rio de Janeiro: GEO UERJO, 2016, p. 26-43.

DE MATTOS, C. Globalización y metropolización en Santiago de Chile: una historia de continuidad y cambios. En: MIDEPLAN y UNIVERSIDAD ALBERTO HURTADO (editores). Metropolización en Chile: interrogantes y desafios. Santiago de Chile: MIDEPLAN, 1999, p. 25-63.

ESCOBAR, A. El lugar de la naturaleza y la naturaleza del lugar: ¿globalización o postdesarrollo? En: LANDER, E. (editor). La colonialidad del saber: eurocentrismo y ciencias sociales, perspectivas latinoamericanas. Buenos Aires: Consejo Latinoamericano de Ciencias Sociales, 2000.

ESCOBAR, A. Culture sits in places: reflections on globalism and subaltern strategies of localization. Political Geography, 2001, Vol. 20, №2, p. 139-174.

EVANS, J. \& JONES, P. The walking interview: Methodology, mobility and place. Applied Geography, 2011, Vol. 31, p. 849-858.

GONZÁLEZ, S. La geografía escalar del capitalismo actual. Scripta Nova. Revista Electrónica de Geografía y Ciencias Sociales, 2005, Vol. IX, N 189. Disponible en Internet: http://www.ub.edu/ geocrit/sn/sn-189.htm

HENRÍQUEZ, C. y ARENAS, F. Algunas claves sobre las transformaciones urbanas de la ciudad media de Los Ángeles. En: BELTRAO, M.; ELIAS, D. \& SOARES, M.C. (editores). Agentes econômicos e reestruturação urbana e regional: Marabá e Los Ángeles. Sao Paulo: Cultura Acadêmica, 2016.

HENRÍQUEZ, C. Modelando el crecimiento de ciudades medias: Hacia un desarrollo urbano sustentable. Santiago de Chile: Ediciones UC, 2014.

HIDALGO, R. La Vivienda social en Chile y la construcción del espacio urbano en el Santiago del siglo XX. EURE, 2002, Vol. 28, № 83, p. 83-106.

HIDALGO, R.; DE MATTOS, C. y ARENAS, F. Chile: Del país urbano al país metropolitano. Santiago de Chile: GEOlibros-Colección EURE-libros, 2009. 
INSTITUTO NACIONAL DE ESTAdístICAS (INE). Censo 2012. Resultados XVIII Censo de Población. Santiago de Chile: INE, 2012.

JIRON, P.; IMILIAN, W. y ITURRA, L. Relearning to travel in Santiago: the importance of mobile place-making and travelling know-how. Cultural Geographies, 2016, Vol. 23, p. 599-614.

JORDAN, R. y SIMIONI, D. Ciudades intermedias de América Latina y el Caribe: propuestas para la gestión urbana. Santiago de Chile: CEPAL, 1998.

KLAUFUS, C. Watching the city grow: remittances and sprawl in intermediate Central American cities. Environment and Urbanization, 2010, Vol. 22, No 1, p. 125-137.

LOMBARD, M. Lugarización y la construcción de asentamientos informales en México. Revista INVI, 2015, Vol. 30, No 83, p. 117-146.

MASSEY, D. Space, Place and Gender. Minneapolis: University of Minnesota Press, 1994.

MASSEY, D. Un sentido global del lugar. En: ALBET, A. y BENACH, N. (editores). Doreen Massey: Un sentido global del lugar. Barcelona: Icaria, 2012, p. 112-129.

MATURANA, F., BELTRÃO SPOSITO, ME., BELLET, C., ARENAS, F. Y HENRÍQUEZ, C. (editores.) "Sistemas urbanos y ciudades medias en Iberoamérica". Serie GeoLibros, Instituto de Geografía, Pontificia Universidad Católica de Chile, Santiago, Chile. 2018

MATURANA, F. y ROJAS, A. (editores). Ciudades intermedias en Chile. Territorios olvidados. Santiago de Chile: RIL Editores, 2015.

MATURANA, F. ¿Ciudad media o ciudad intermedia? Evolución conceptual y estudio en Chile. En: MATURANA, F. y ROJAS, A. (editores). Ciudades intermedias en Chile. Territorios olvidados. Santiago de Chile: RIL Editores, 2015, p. 21-42.

NEWING, H.; EAGLE, C.M. y PURI, R.K. Conducting Research in Conservation: Social Science Methods and Practice. London/New York: Routledge, 2010.

PORTES, A. y ROBERTS, B.R. The free-market city: Latin American urbanization in the years of the neoliberal experiment. Studies in Comparative International Development, 2005, Vol. 40, No 1, p. 43-82.

RICKETTS, J.; EVANS, J. \& JONES, P. Mobile methodologies: Theory, technology and practice. Geography Compass, 2008, Vol. 2, N 5, p. 1266-1285.

ROBERTS, B. Managing Systems of Secondary Cities. Bruselas: Cities Alliance/UNOPS, 2014.

RODRIGUEZ, J. y GONZÁLEZ, D. Redistribución de la población y migración interna en Chile: continuidad y cambio según los últimos cuatro censos nacionales de población y vivienda. Revista de Geografía Norte Grande, 2006, Vol. 35, p. 7-28. 
ROJAS, A.; MATURANA, F. y MORALES, M. Evolución histórica de las ciudades intermedias en el siglo XX: Crecimiento, Jerarquía y funcionalidad. En En: MATURANA, F. y ROJAS, A. (editores). Ciudades intermedias en Chile. Territorios olvidados. Santiago de Chile: RIL Editores, 2015, p. 43-74.

SALAZAR, G.; FONK, M. y IRARRAZABAL, F. Paisajes en movimiento: sentidos de lugar y prácticas interculturales en ciudades de la Región de La Araucanía. Revista Chungará, 2017a, Vol. 49, № 2, p. 251-264.

SALAZAR, G.; IRARRAZABAL, F. \& FONK, M. Exploring intermediate cities in Latin America: mixed mobile methods for mobility assessment in Villarrica, Chile. The Geographical Journal. Vol. 138:3 p247-260 doi: 10.1111/geoj.12210 (2017b).

SHELLER, M. \& URRY, J. The new mobilities paradigm. Environment and planning A, 2006, Vol. 38, $N^{\circ} 2$, p. 207-226.

SKEWES, J.; REHBEIN, R. y MANCILLA, C. Ciudadanía y sustentabilidad ambiental en la ciudad: la recuperación del humedal Angachilla y la organización local en la Villa Claro de Luna, Valdivia, Chile. EURE, 2012, Vol. 38, № 12, p. 127-145.

UNITED NATIONS FUND FOR POPULATION ACTIVITIES (UNFPA). State of World Population 2007: Unleashing the Potential of Urban Growth. New York: UNFPA, 2007.

UN-HABITAT. State of the World's Cities 2010/2011: bridging the urban divide. London: Earthscan, 2010.

ZUNINO, H.M. y HIDALGO, R. En busca de una utopía verde: migrantes de amenidad en la Comuna de Pucón, IX región de la Araucanía, Chile. Scripta Nova. Revista Electrónica de Geografía y Ciencias Sociales, 2010, Vol. XIV, N 331. Disponible en Internet: http://www.ub.edu/geocrit/sn/sn-331/ sn-331-75.htm 